\title{
Safety and Use of Complementary and Alternative Medicine in Norway During the First Wave of the COVID-19 Pandemic Using an Adapted Version of the I-CAM-Q: A Cross-Sectional Survey
}

Agnete Egilsdatter Kristoffersen ( $\sim$ agnete.kristoffersen@uit.no )

UiT The Arctic University of Norway

Miek C. Jong

UiT The Arctic University of Norway

Johanna Hök Nordberg

Karolinska Institutet

Esther T. van der Werf

Louis Bolk Institute

Trine Stub

UiT The Arctic University of Norway

\section{Research Article}

Keywords: CAM, T\&CM, COVID-19, Norway, Complementary therapies, safety

Posted Date: June 18th, 2021

DOI: https://doi.org/10.21203/rs.3.rs-612371/v1

License: (c) (1) This work is licensed under a Creative Commons Attribution 4.0 International License.

Read Full License 


\section{Abstract}

\section{Background}

The COVID-19 pandemic lockdown has had a profound impact on physical and mental well-being throughout the world. Previous studies have revealed that complementary and alternative medicine (CAM) is frequently used for, and can be potential beneficial for strengthening physical mental resilience. The aim of this study was therefore to determine the prevalence, associations and reasons for use of CAM during the first wave of the COVID-19 pandemic among a representative sample of the Norwegian population, and determine possible effects and adverse effects of the treatments.

\section{Methods}

Computer assisted telephone interviews using a COVID-adapted I-CAM-Q questionnaire were conducted with 1008 randomly selected Norwegians aged 16 and above using multistage sampling during April and May 2020 applying age and sex quotas for each area. Descriptive statistics were carried out using Pearson's Chi-square test, Fisher exact test, and Independent samples T-tests to identify group differences.

\section{Results}

The study revealed that two thirds of the respondents (67\%) had used CAM within the first three months of the COVID-19 pandemic, in particular CAM modalities that did not involve a provider. Most used were natural remedies and dietary supplement (57\%, mainly vitamins and minerals), but self-help practices like yoga and meditation were also widely used (24\%). Women used CAM modalities significantly more than men $(77 \%$ vs. $58 \%)$. Most of the respondents found the modalities they used beneficial, and $5 \%$ reported adverse effects of the treatments.

\section{Conclusions}

The first wave of the COVID-19 pandemic has not profoundly changed the overall need of Norwegian citizens to seek care outside the conventional healthcare system, but rather caused a shift in the type of CAM modalities used. The risk profile of these modalities was generally low as only $5 \%$ of the respondents reported adverse effects. Further studies are needed to confirm the change from provider based to self-administered CAM use.

\section{Background}

The COVID-19 pandemic lockdown has a profound impact on physical and mental well-being throughout the world [1]. In Norway, a nationwide lockdown was implemented on March 12, 2020 [4]. Kindergarten, schools, and universities were closed and employees who could work from home were instructed to do so. Although a lockdown most likely decreased the spread and harm from COVID-19 [2], studies describe that such restrictions might have serious mental health effects on the population [5-8], in particular in 
groups that already were vulnerable [9]. While the COVID-19 pandemic has led to increased activity for parts of health care, other parts were significantly reduced: Consultations and operations have been postponed due to infection control and health care providers in quarantine [3], and referral to a wide range of mental health services has decreased [1]. Health care providers working outside established health care services were instructed to close down their clinics and stop offering treatments that included physical contact between provider and client. This led to a lockdown among many complementary and alternative medicine (CAM) providers or reorganisation of their practice to telephone- and video consultations [4] where that could be of help to their clients.

CAM is defined as a group of diverse medical and health care practices and products that are not generally considered part of conventional medicine [5]. Several subcategories of health care-seeking behaviour fall under the definition of CAM: Visits to CAM providers; use of natural remedies and dietary supplements; and different types of self-help practices [6]. CAM is widely used, ranging from $10 \%$ [7] to $76 \%$ worldwide [8]. CAM is also commonly used in Europe [7], and Scandinavia [9, 10]. In Norway a recently published study demonstrated that $62.2 \%$ of the respondents reported to have used CAM within a 12 months period [11]. Most respondents had used natural remedies and dietary supplements (47.7\%), followed by self-help practices such as yoga and meditation (29.1\%), and consultations with CAM providers $(14.7 \%)[11]$.

A previous literature review described that CAM is frequently used to strengthen physical and mental resilience, and describe a possibly direct effect of CAM approaches like vitamin A, D, and mind-body interventions on immune functions and influenza, relevant for people during the COVID-19 pandemic [12]. However, it is not known whether people in Norway are making use of CAM to deal with possible negative health effects of the COVID-19 related restrictions such as weight gain [21], depression, anxieties, economic pressure, and mental illness [22]. Furthermore, people access to CAM might have been limited as the CAM providers had to close down their practice during the lockdown the first three months of the pandemic.

The aim of this study was therefore to determine the prevalence, associations, and reasons for use of CAM during the first wave of the COVID-19 pandemic among a representative sample of the Norwegian population, and further determine possible effects and adverse effects of the modalities.

\section{Methods}

In Norway, a national cross-sectional survey was carried out between April 28 and May 52020 using computer-assisted telephone interviews. The survey was conducted in collaboration with the marketing research company Ipsos A/S and had a target sample of 1000 people, representing the Norwegian population of 5.4 million inhabitants [13]. The sample was stratified by sex, age and region of residence and drawn from Norwegian residents aged 16 and above living in private households with a landline telephone or a cell phone using random quota sampling. Up to 7 attempts were made to reach the selected person. $\mathrm{N}=4,337$ were unreachable after 7 calling attempts (figure 1 ). Individuals who were 
reached and refused participation $(n=1,881)$ were considered non-respondents, leading to a response rate of $34.5 \%$. The final sample contained 1008 individuals, 487 women and 521 men (figure 1).

\section{Survey instrument}

A COVID-19 adapted version of the International Questionnaire to Measure Use of Complementary and Alternative Medicine (I-CAM-Q) was used [11, 14] addressing COVID-19 related use in addition to use for acute illness, chronic illness, well-being and other, unspecified reasons. The survey consisted of three parts: The first part related to CAM use offered by CAM providers, the second part included questions regarding use of natural remedies and dietary supplements while the third part included questions related to self-help practices such as mindfulness, yoga, and relaxation techniques. In all parts, the questions referred to use during the last three months. Demographic characteristics collected were sex, age, level of education, household income and place of residence. All data were anonymously collected and reported.

\section{Measures}

\section{Measures of personal characteristics}

Age was obtained as an open question and assessed as a continuous variable as well as categorical after being devided into four groups; $16-24$ years; $25-39 ; 40-59$ years; and 60 year or more.

Level of education was collected using six categories: 1. Primary school up to 8 years; 2 . primary school up to 10 years; 3 . secondary school; 4 . college/university less than 4 years; and 5 . college/university 4 years or more. These were merged into primary school (1-2); secondary school (3); and college/university (4-5).

Household income was collected using the following categories (NOK $<100,000,100,000-199,000$, 200,000-299,000, 300,000-399,000. 400,000-499,000, 500,000-599,000, 600,000-799,000, 800,000$999,000,1000,000-1500,000$ and more than NOK 1500,000). These categories were merged into low household income (<NOK400,000), middle household income (NOK 400,000-799,000) and high household income (NOK 800,000 or more).

Other personal characteristics included sex (female, male) and residence (merged into the Norwegian regions South-East, South, West, Mid (Trøndelag), and North).

\section{CAM providers}

The measure consultations with CAM providers were specified by visits to massage therapists, naprapaths, acupuncturists, coaches, healers, homeopaths, cupping therapists, herbalists, traditional healers, osteopaths, kinesiologists, and other CAM providers. 
The respondents were asked whether they had consulted one or more CAM providers and if yes; reasons, benefit, and possible adverse effects of the treatments.

\section{Natural remedies and dietary supplements}

Use of natural remedies and dietary supplements were mapped by providing the respondents with a list of remedies organized as herbs (ginger, curcumin, garlic, green tea, herbal tea, cranberry, blueberry/blueberry extract, oregano, echinacea, chaga, and other), vitamins and minerals (vitamin $\mathrm{B}$, vitamin $\mathrm{C}$, vitamin D, magnesium, calcium, iron, zinc, selenium, and other vitamins and minerals), homeopathic remedies, Bach flower remedies, and dietary supplements (Omega 3, 6 or 9, protein shake, Q10, and other dietary supplements). The respondents were asked whether they had used one or more of these remedies, and if yes; reasons, benefit, and possible adverse effects of this use.

\section{Self-help practices}

Use of self-help practices were measured by specific questions regarding the use of meditation, yoga, qigong, tai chi, relaxation, visualization, mindfulness, lightning process, neuro-linguistic programming (NLP), participation in traditional healing rituals, and other self-help practices. The respondents were asked if they had used these self-help practices and further; why, whether they found it to be beneficial, and if they experienced adverse effects.

\section{Over-all use of CAM}

Over-all-CAM use was measured by calculating the total number of CAM users reported, combining the variables modalities provided by CAM providers, natural remedies and dietary supplements, and self-help practices.

\section{Statistics}

Descriptive statistics were carried out using Statistical Package for Social Sciences (SPSS) v. 26.0. Pearson's chi-square tests, Fisher exact tests, and T-test were performed to identify differences in sociodemographic factors (age, education level, household income) between men and women and between CAM users and non-CAM users.

\section{Results}

\section{Basic characteristics of the respondents}

The survey consisted of slightly more men than women ( $51.7 \%$ vs $48.3 \%)$, with a mean age of 44.95 and 48.03 years respectively $(p=0.009)$. The majority of respondents had high income $(41.7 \%)$, a college or 
university education (53.5\%) and were living in the South-Eastern part of Norway (50.7\%). The men had higher income $(p=0.017)$ and lower education $(p=0.002)$ compared to women (table 1$)$.

Table 1. Basic characteristics of the respondents 


\begin{tabular}{|c|c|c|c|c|c|c|c|}
\hline & \multicolumn{2}{|l|}{ Total } & \multicolumn{2}{|c|}{ Women } & \multicolumn{3}{|l|}{ Men } \\
\hline & $\%$ & $(n=1008)$ & $\%$ & $(n=487)$ & $\%$ & $(n=521)$ & $\mathrm{p}$-value \\
\hline \multicolumn{8}{|l|}{ Gender } \\
\hline Men & 51.7 & $(521)$ & & & & & \\
\hline Women & 48.3 & $(487)$ & & & & & \\
\hline \multicolumn{8}{|l|}{ Age } \\
\hline Mean age (years) & 46.44 & (1008) & 48.03 & & 44.95 & & $0.009^{2}$ \\
\hline (SD) & \multicolumn{2}{|c|}{$(18.678)$} & \multicolumn{2}{|c|}{$(18.959)$} & \multicolumn{2}{|c|}{ (18.305) } & \\
\hline $18-24$ years & 15.9 & $(160)$ & 14.8 & $(72)$ & 16.9 & (88) & $0.059^{1}$ \\
\hline 25-39 years & 25.1 & $(253)$ & 22.0 & $(107)$ & 28.0 & $(146)$ & \\
\hline $40-59$ & 32.7 & $(330)$ & 34.4 & $(168)$ & 31.1 & $(162)$ & \\
\hline$\geq 60$ years & 26.3 & $(265)$ & 28.7 & $(140)$ & 24.0 & (125) & \\
\hline Household income ${ }^{3}$ & & & & & & & $0.017^{1}$ \\
\hline Low & 10.5 & $(106)$ & 11.5 & $(56)$ & 9.6 & $(50)$ & \\
\hline Middle & 26.8 & $(270)$ & 25.5 & $(124)$ & 28.0 & $(146)$ & \\
\hline High & 41.7 & $(420)$ & 38.4 & $(187)$ & 44.7 & $(233)$ & \\
\hline Do not want to answer & 6.2 & (62) & 6.2 & (30) & 6.1 & (32) & \\
\hline Do not know & 14.9 & $(150)$ & 18.5 & (90) & 11.5 & $(60)$ & \\
\hline Years of Education & & & & & & & $0.002^{1}$ \\
\hline Primary school & 11.0 & (111) & 11.1 & (54) & 10.9 & (57) & \\
\hline Secondary school & 35.5 & (358) & 30.2 & $(147)$ & 40.5 & $(211)$ & \\
\hline College/university & 53.5 & (539) & 58.7 & $(286)$ & 48.6 & $(253)$ & \\
\hline Region of residence & & & & & & & $0.929^{1}$ \\
\hline South-East & 50.7 & $(511)$ & 51.1 & $(249)$ & 50.3 & $(262)$ & \\
\hline South & 5.7 & (57) & 5.3 & $(26)$ & 6.0 & (31) & \\
\hline West & 25.3 & $(255)$ & 26.1 & $(127)$ & 24.6 & (128) & \\
\hline Mid (Trøndelag) & 8.7 & (88) & 8.4 & $(41)$ & 9.0 & (47) & \\
\hline North & 9.6 & (97) & 9.0 & (44) & 10.2 & (53) & \\
\hline
\end{tabular}


${ }^{1}$ Pearson chi-square test; ${ }^{2}$ Independent samples t-test; ${ }^{3}$ Low (<NOK400,000), middle (NOK 400,000$799,000)$, and high ( $\geq$ NOK 800,000).

\section{CAM use}

A total of $67.2 \%$ of the respondents had used CAM during the first three months of the COVID-19 pandemic, more women $(77.4 \%)$ than men $(57.6 \%, p<0.001$, table 2$)$. Less than $8 \%$ of the survey respondents-had consulted CAM providers (table 2). Most frequently used were natural remedies and dietary supplements (57\%), followed by self-help practises (24.2\%).

Table 2. Use of CAM among female and male respondents during the first three months of the COVID-19 pandemic

\begin{tabular}{|c|c|c|c|c|}
\hline & $\begin{array}{l}\text { Total } \\
\%(n)\end{array}$ & $\begin{array}{c}\text { Women } \\
\%(n)\end{array}$ & $\begin{array}{l}\text { Men } \\
\%(n)\end{array}$ & p-value \\
\hline Consultations with CAM providers & $7.8(79)$ & $10.1(49)$ & $5.5(30)$ & $0.011^{1}$ \\
\hline Use of natural remedies and dietary supplements & $57.0(575)$ & $66.7(325)$ & $48.0(250)$ & $<0.001^{1}$ \\
\hline Herbs & $14.9(150)$ & $19.1(93)$ & $10.9(57)$ & $<0.001^{1}$ \\
\hline Vit amins and minerals ${ }^{3}$ & $43.1(434)$ & $52.4(255)$ & $34.4(179)$ & $<0.001^{1}$ \\
\hline Homeopatic remedies & $1.3(13)$ & $1.0(5)$ & $1.5(8)$ & $0.474^{1}$ \\
\hline Bach flower remedies & $0.4(4)$ & $0.8(4)$ & $0.0(0)$ & $0.054^{2}$ \\
\hline Other dietary supplements & $23.7(239)$ & 26.5 (129) & $21.1(110)$ & $0.045^{1}$ \\
\hline Use of self-help practices ( yoga, meditation etc.) & $24.2(244)$ & 32.4 (158) & $16.5(86)$ & $<0.001^{1}$ \\
\hline Total use of CAM (providers, supplements or self-help) & $67.2(677)$ & $77.4(4)$ & $57.6(300)$ & $<0.001^{1}$ \\
\hline
\end{tabular}

1 Pearson Chi-Square test; 2 Fisher exact test; ${ }^{3}$ Vit amins and minerals other than multi-vitamin tablets/mixture

\section{Consultation with CAM providers}

CAM providers were consulted by $7.8 \%$ of the respondents, $10.1 \%$ of the women and $5.5 \%$ of the men $(p=0.011)$ during the first three months of the COVID-19 pandemic (table 2). Massage therapists $(3 \%)$, naprapaths $(1.5 \%)$, psychotherapists $(1.4 \%)$, acupuncturists $(1.2 \%)$, and reflexologists $(1 \%)$ were the most visited CAM providers. Most of the respondents experienced positive effect of the treatments, ranging from $91.7 \%$ (acupuncturist) to $100 \%$ (naprapaths, psychotherapists, and reflexologists) mainly for chronic complaints. One had consulted a traditional healer for preventing COVID-19. Men and women consulted CAM providers to a similar degree except for massage therapists who were more frequently visited by women $(4.1 \%)$ compared to men $(1.9 \%, p=0.041$, table 3$)$.

The respondents who had consulted CAM providers were mainly middle aged, with secondary education or lower university education, high income, and living in the South-Eastern part of Norway. They did not differ from respondents not consulting CAM providers in regard to these factors (table 4, $p>0.217$ ). Similar characteristics were found for both women and men apart from educational level were the women who consulted CAM providers were more likely to have college or university education $(61.2 \%)$ 
compared to men $(23.3 \%, p=0.001$, table 5$)$. Men consulting CAM providers differed in this regard from men who did not where $50.1 \%$ reported to have college or university education $(p=0.007)$. Women, on the other hand had similar education as those who did not consult CAM providers $(p=0.931)$.

Table 3. The most commonly used CAM modalities during the first three months of the COVID-19 pandemic, reason for use and self-perceived benefit

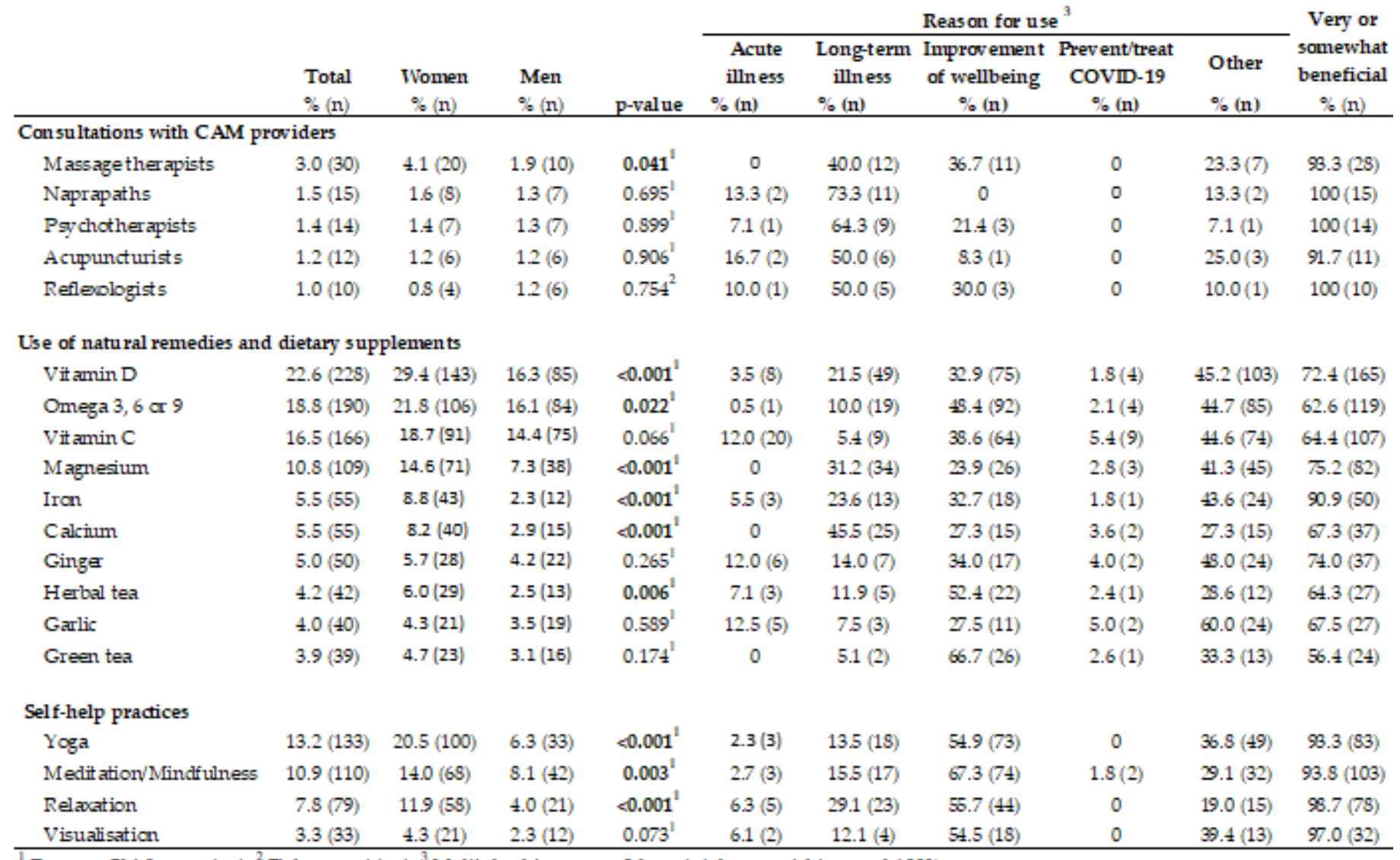

${ }^{1}$ Pearscn Chi-Square test; ${ }^{2}$ Fisher exact test; ${ }^{3}$ Multiple chioces possible so total sum might exceed $100 \%$

\section{Natural remedies and dietary supplements}

More than half of the respondents (57\%) had used natural remedies and dietary supplements during the first three months of the COVID-19 pandemic (table 2), mostly reported to be beneficial, ranging from $56.4 \%$ (green tea) to $90.9 \%$ (iron). Natural remedies and dietary supplements were mainly used for wellbeing or other reasons, only $1.5 \%(n=15)$ used natural remedies and dietary supplements to treat or prevent COVID-19; vitamin $C(n=9)$, vitamin $D(n=4)$, Omega 3,6 or 9 fatty acid $(n=4)$, and magnesium $(n=3)$. Vitamin D (22.6\%), Omega 3.6 or $9(18.8 \%)$, vitamin $C(16.5 \%)$, magnesium (10.8\%), iron (5.5\%), calcium (5.5\%), ginger (5.0\%), herbal tea (4.2\%), garlic (4.0\%), and green tea (3.9\%) were most used (table 3). Vitamin $D$, omega 3,6 , and 9 , magnesium, iron, calcium, and herbal tea were most frequently used by women $(p<0.023$, table 3$)$. Women used significantly more natural remedies and dietary supplements compared to men $(66.7 \%$ vs. $48.0 \%, p<0.001)$. Besides from being female, the users of natural remedies and dietary supplements were older than the non-users ( 47.5 years vs. 45.0 years, $p=0.035$ ), and with 
higher age among the female users (48.8 compared to 45.8 years among male users, $p=0.063$ ).

Education and household income were similar among users and non-users of natural remedies and dietary supplements (table 4, p>0.067), but women were more likely to have college or university education compared to the male users of natural remedies and dietary supplements $(60.3 \%$ vs $45.0 \%$, $p=0.001$, table 5). Neither women nor men differed from the non-users of natural remedies and dietary supplements in regard to education.

\section{Self-help practices}

Self-help practices were used by $24.2 \%(n=244)$ of the respondents during the first three months of the COVID-19 pandemic, the majority with positive effect ranging from $93.3 \%$ (yoga) to $98.7 \%$ (relaxation). The self-help practices were mainly used to improve well-being, only 3 (2 meditation/mindfulness and 1 Qigong) had used this to prevent or treat COVID-19. Twice as many women (32.4\%) than men used selfhelp practices $(16.5 \%, \mathrm{p}<0.001$, table 2$)$. The most frequently used self-help practice among women was yoga (20.5\%), while meditation/mindfulness (8.1\%) were the most used self-help practice among men (table 3). The respondents practicing self-help practices were younger, and-more likely to have a college/university education compared to those who did not partake in such practices (table 4). Women reporting self-help practices were more likely to have university education ( $69 \%$ vs. $53.8 \%, p=0.003)$ than women who did not. Men also, but not at a significant level $(51.2 \%$ vs. $48 \%, p=0.793)$.

Table 4. Associations for use of CAM during the first three months of the COVID-19 pandemic

\begin{tabular}{|c|c|c|c|c|c|c|c|c|c|c|c|c|}
\hline & \multirow{2}{*}{\multicolumn{6}{|c|}{ Natural remedies and dietary }} & \multirow{2}{*}{\multicolumn{3}{|c|}{ Self-help practices }} & \multirow{2}{*}{\multicolumn{3}{|c|}{ Use of any CAM modality }} \\
\hline & & & & & & & & & & & & \\
\hline & $\begin{array}{l}\text { Yes } \\
\% \text { (n) }\end{array}$ & $\begin{array}{c}\text { No } \\
\%(n)\end{array}$ & pevalue & $\begin{array}{l}\text { Yes } \\
\% \text { (n) }\end{array}$ & $\begin{array}{c}\text { No } \\
\% \text { (n) }\end{array}$ & peralue & $\begin{array}{c}\text { Yes } \\
\%(n)\end{array}$ & $\begin{array}{c}\text { No } \\
\%(n)\end{array}$ & pevalue & $\begin{array}{l}\text { Yes } \\
\%(n)\end{array}$ & $\begin{array}{c}\text { No } \\
\%(n)\end{array}$ & p-value \\
\hline Sex & & & $0.011^{1}$ & & & $<0.001^{1}$ & & & $<0.001^{1}$ & & & $<0.001^{1}$ \\
\hline Female & $62.0(49)$ & $47.1(348)$ & & $56.5(325)$ & $37.4(162)$ & & $64.8(158)$ & $43.1(329)$ & & $55.7(377)$ & $332(110)$ & \\
\hline Male & $38.0(30)$ & $52.9(491)$ & & $43.5(250)$ & $62.6(271)$ & & $35.2(86)$ & $56.9(435)$ & & $44.3(300)$ & $66.8(221)$ & \\
\hline Age (y ears) & & & $0.302^{1}$ & & & $0.068^{1}$ & & & $<0.001^{1}$ & & & 0.324 \\
\hline $16-24$ & $8.9(7)$ & $16.5(153)$ & & $15.8(91)$ & $15.9(69)$ & & $20.9(51)$ & $14.3(109)$ & & $16.5(112)$ & $14.5(48)$ & \\
\hline 25.39 & $29.23(23)$ & $24.8(230)$ & & 23.5 (135) & $27.3(118)$ & & $32.8(80)$ & $22.6(173)$ & & $25.4(172)$ & $24.5(81)$ & \\
\hline $40-59$ & $36.7(29)$ & $32.4(301)$ & & $31.3(180)$ & $34.6(150)$ & & $34.0(83)$ & $32.3(247)$ & & $30.9(209)$ & $36.6(121)$ & \\
\hline $60+$ & $25.3(20)$ & $26.4(245)$ & & $29.4(169)$ & $22.2(96)$ & & $12.3(30)$ & $30.8(235)$ & & $27.2(184)$ & $24.5(81)$ & \\
\hline Education & & & $0.218^{1}$ & & & $0.652^{1}$ & & & $0.001^{1}$ & & & 0.179 \\
\hline Primary/Second ar & $53.2(42)$ & $46.0(427)$ & & $45.9(264)$ & $47.3(205)$ & & $37.3(91)$ & $49.5(378)$ & & $45.1(305)$ & $49.5(164)$ & \\
\hline College/University & $46.8(37)$ & $54.0(502)$ & & $54.1(311)$ & $52.7(228)$ & & 62.7 (153) & $50.5(386)$ & & $54.9(372)$ & $50-5(167)$ & \\
\hline Household income & & & $0.726^{1}$ & & & $0.132^{\prime}$ & & & $0.489^{\prime}$ & & & 0.249 \\
\hline Low & $15.9(10)$ & $13.1(96)$ & & $15.3(70)$ & $10.7(36)$ & & $15.7(31)$ & $12.5(75)$ & & $14.7(79)$ & $10.4(27)$ & \\
\hline Middel & $30.2(19)$ & $34.2(251)$ & & $34.1(156)$ & 33.7 (114) & & $31.8(63)$ & $34.6(207)$ & & $33.3(179)$ & 35.1 (91) & \\
\hline High & $540(34)$ & $52.7(386)$ & & $50.7(232)$ & 55.6 (188) & & $52.5(104)$ & $52.8(316)$ & & $52.0(279)$ & 54.4 (141) & \\
\hline Region of residence & & & $0.556^{2}$ & & & $0.563^{1}$ & & & $0.157^{1}$ & & & 0.518 \\
\hline East & $58.2(46)$ & $50.1(465)$ & & $51.5(29)$ & $49.7(215)$ & & $54.5(133)$ & $49.5(378)$ & & $52.1(353)$ & $47.7(158)$ & \\
\hline South & $2.5(2)$ & $5.9(55)$ & & $5.2(30)$ & $62(27)$ & & $4.5(11)$ & $6.0(46)$ & & $5.0(34)$ & $6.9(23)$ & \\
\hline West & $20.3(16)$ & $25.7(239)$ & & $26.4(152)$ & $23.8(103)$ & & $20.1(49)$ & $27.0(206)$ & & $25.3(171)$ & $25.4(84)$ & \\
\hline Mid (Trondelag) & $8.9(7)$ & $8.7(81)$ & & $8.2(47)$ & $9.5(41)$ & & $10.7(26)$ & $8.1(62)$ & & $8.1(55)$ & $10.0(33)$ & \\
\hline Narth & $10.1(8)$ & $9.6(39)$ & & $8.7(50)$ & $10.9(47)$ & & $10.2(25)$ & $9.4(72)$ & & $9.5(64)$ & $10.0(33)$ & \\
\hline
\end{tabular}

${ }^{1}$ Pearscn chi-square test; ${ }^{2}$ Fisher exact test

Table 5. Gender differences in associations for use of CAM during the first three months of the COVID-19 
pandemic

\begin{tabular}{|c|c|c|c|c|c|c|c|c|c|c|c|c|c|c|c|c|}
\hline & \multicolumn{4}{|c|}{ CAM providers } & \multicolumn{4}{|c|}{ Natural remedies and dietary supplements } & \multicolumn{4}{|c|}{ Self-help practices } & \multicolumn{4}{|c|}{ Total use of CAM } \\
\hline & Total & $\begin{array}{c}\text { Women } \\
\%(n)\end{array}$ & $\begin{array}{l}\text { Men } \\
\% \text { (n) }\end{array}$ & pwalue & Total & $\begin{array}{c}\text { Women } \\
\%(n)\end{array}$ & $\begin{array}{l}\text { Men } \\
\% \text { (n) }\end{array}$ & pwalue & Total & $\begin{array}{c}\text { Women } \\
\% \text { (n) }\end{array}$ & $\begin{array}{l}\text { Men } \\
\% \text { (n) }\end{array}$ & pevalue & Total & $\begin{array}{c}\text { Women } \\
\% \text { (n) }\end{array}$ & $\begin{array}{l}\text { Men } \\
\% \text { (n) }\end{array}$ & povalue \\
\hline Age (y ears) & & & & $0.650^{2}$ & & & & $0.152^{1}$ & & & & $0.010^{1}$ & & & & $0.103^{1}$ \\
\hline $16-24$ & $8.9(7)$ & $8.2(4)$ & $10.0(3)$ & & $15.8(91)$ & $13.8(45)$ & $18.4(46)$ & & $20.9(51)$ & $19.0(30)$ & $24.4(21)$ & & 16.5 & $15.1(57)$ & $18.3(55)$ & \\
\hline $25-39$ & $29.23(23)$ & $24.5(12)$ & 36.7 (11) & & 23.5 (135) & $21.5(70)$ & $26.0(65)$ & & $32.8(80)$ & $27.8(44)$ & $41.9(36)$ & & $25.4(172)$ & $22.8(86)$ & $28.7(86)$ & \\
\hline 40.59 & $36.7(29)$ & $40.8(20)$ & $30.0(9)$ & & $31.3(180)$ & $34.2(111)$ & $27.6(69)$ & & $34.0(83)$ & $36.7(58)$ & $29.1(25)$ & & $30.9(209)$ & $34.0(128)$ & $27.0(81)$ & \\
\hline $60+$ & $25.3(20)$ & $26.5(13)$ & $23.3(7)$ & & $29.4(169)$ & $30.5(99)$ & $28.0(70)$ & & $12.3(30)$ & $16.5(26)$ & $4.7(4)$ & & $27.2(184)$ & $28.1(106)$ & $26.0(78)$ & \\
\hline Education & & & & $0.001^{1}$ & & & & $0.001^{1}$ & & & & $0.006^{2}$ & & & & $<0.001^{1}$ \\
\hline Primary/Secondar & $53.2(42)$ & $38.8(19)$ & $76.7(23)$ & & $45.9(264)$ & 39.7 (129) & $540(135)$ & & $49.5(378)$ & $46.2(152)$ & $52.0(226)$ & & $45.1(305)$ & $38.5(145)$ & $53.3(160)$ & \\
\hline College/University & $46.8(37)$ & $61.2(30)$ & $23.3(27)$ & & $54.1(311)$ & $60.3(196)$ & $46.0(115)$ & & $50.5(386)$ & $53.8(177)$ & $48.0(209)$ & & $54.9(372)$ & $61.5(232)$ & $46.7(140)$ & \\
\hline Household income & & & & $0.342^{2}$ & & & & $0.703^{1}$ & & & & $0.416^{1}$ & & & & $0.587^{1}$ \\
\hline Low & $15.9(10)$ & $20.0(8)$ & $8.7(2)$ & & $15.3(70)$ & $16.5(41)$ & $13.9(29)$ & & $15.7(31)$ & $18.0(24)$ & $10.8(7)$ & & $14.7(79)$ & $16.2(47)$ & $13.0(32)$ & \\
\hline Middel & $30.2(19)$ & $25.0(10)$ & $39.1(9)$ & & 34.1 (156) & $32.9(82)$ & $35.4(74)$ & & $31.8(63)$ & $30.8(41)$ & $33.8(22)$ & & $33.3(179)$ & $33.0(96)$ & $33.7(83)$ & \\
\hline High & $54.0(34)$ & $55.0(22)$ & $52.2(12)$ & & $50.7(232)$ & $50.6(126)$ & $50.7(106)$ & & $52.5(104)$ & $51.1(68)$ & $55.4(36)$ & & $52.0(279)$ & $50.9(148)$ & $53.3(131)$ & \\
\hline Region of residence & & & & $0.213^{2}$ & & & & $0.614^{1}$ & & & & $0.365^{2}$ & & & & $0.477^{1}$ \\
\hline East & $58.2(46)$ & $63.3(31)$ & $50.0(15)$ & & $51.5(296)$ & $52.0(169)$ & $50.8(127)$ & & 54.5 (133) & $57.0(90)$ & $50.0(43)$ & & 52.1 (353) & $53.3(201)$ & $50.7(152)$ & \\
\hline South & $2.5(2)$ & $2.0(1)$ & $3.3(1)$ & & $5.2(30)$ & $4.0(13)$ & $6.8(17)$ & & 4.5 (11) & $2.5(4)$ & $8.1(7)$ & & $5.0(34)$ & $3.7(14)$ & $6.7(20)$ & \\
\hline West & $20.3(16)$ & $20.4(10)$ & $20.0(6)$ & & $26.4(152)$ & $27.4(89)$ & $25.2(63)$ & & $20.1(49)$ & $19.6(31)$ & $20.9(18)$ & & $25.3(171)$ & $26.0(98)$ & $24.3(73)$ & \\
\hline Mid (Trondelag) & $8.9(7)$ & $10.2(5)$ & $6.7(2)$ & & $8.2(47)$ & $7.7(25)$ & $8.8(22)$ & & $10.7(26)$ & $10.8(17)$ & $10.5(9)$ & & $8.1(55)$ & $8.0(30)$ & $8.3(25)$ & \\
\hline North & $10.1(8)$ & $4.1(2)$ & $20.0(6)$ & & $8.7(50)$ & $8.9(29)$ & $8.4(21)$ & & $10.2(25)$ & $10.1(15)$ & $10.5(9)$ & & $9.5(64)$ & $9.0(34)$ & $10.0(30)$ & \\
\hline
\end{tabular}

\section{Safety of CAM}

Most of the respondents (95\%) used CAM without experiencing adverse effects of the treatment. Five percent $(n=34)$, however reported a total of 42 adverse effects. Four of the respondents $(n=4)$ experienced adverse effects after consultations with CAM providers; 2 from acupuncture, 1 from traditional healing and 1 from cupping.

Twenty-one $(n=21)$ respondents reported 27 adverse effects from natural remedies and dietary supplements; 4 from garlic, 4 from iron, 4 from Omega 3,6 or 9, 2 from protein shake, 2 from magnesium, 2 from ginger, 2 from high doses of vitamin C, 2 from zinc, 1 from vitamin $D, 1$ from vitamin $B, 1$ from herbal tea, 1 from lemon, and 1 from calcium.

Finally, eleven ( $n=11)$ respondents report 11 adverse effects from self-help practices: 9 from yoga, 1 from meditation, and 1 from relaxation (table 6 ). In 32 of the 42 cases (76.2\%) adverse effects were reported even though the respondents found the modalities to be very or somewhat beneficial (table 6).

\section{Table 6. Safety and perceived utility reported by respondents}




\begin{tabular}{|c|c|c|}
\hline Modalities & $\begin{array}{c}\text { Number of adverse } \\
\text { effects reported } \\
(n=42)\end{array}$ & Percived utility \\
\hline Modalities recieved from CAM providers & 4 & \\
\hline Acupuncture & 1 & Very beneficial \\
\hline Acupuncture & 1 & Not beneficial \\
\hline Tr aditional healing & 1 & Not beneficial \\
\hline Cupping & 1 & Not beneficial \\
\hline Natural reme dies and dietary suplements & 27 & \\
\hline Garlic & 3 & Very beneficial \\
\hline Garlic & 1 & Somewhat beneficial \\
\hline Iran & 3 & Very beneficial \\
\hline Iran & 1 & Somewhat beneficial \\
\hline Omega 3,6 ar 9 & 1 & Very beneficial \\
\hline Omega 3,6 ar 9 & 1 & Somewhat beneficial \\
\hline Omega 3,6 ar 9 & 2 & Dan't know \\
\hline Protein shake & 1 & Very beneficial \\
\hline Protein shake & 1 & Somewhat beneficial \\
\hline Magnecium & 1 & Very beneficial \\
\hline Magnesium & 1 & Somewhat beneficial \\
\hline Ginger & 1 & Very beneficial \\
\hline Ginger & 1 & Somewhat beneficial \\
\hline High doses vitamin C & 1 & Very beneficial \\
\hline High doses vitamin $\mathrm{C}$ & 1 & Dan't know \\
\hline Zink & 1 & Very beneficial \\
\hline Zink & 1 & Dan't know \\
\hline Vit amin D & 1 & Somewhat beneficial \\
\hline Vitamin B & 1 & Somewhat beneficial \\
\hline Herbal tea & 1 & Very beneficial \\
\hline Lemon & 1 & Very beneficial \\
\hline Calsium tablets & 1 & Dan't know \\
\hline Self-help practices & 11 & \\
\hline Yoga & 4 & Very beneficial \\
\hline Yoga & 4 & Somewhat beneficial \\
\hline Yoga & 1 & Not beneficial \\
\hline Meditation & 1 & Very beneficial \\
\hline Relaxation & 1 & Very beneficial \\
\hline
\end{tabular}

\section{Discussion}

The current study suggests that two out of three Norwegians (67.2\%) used CAM during the first three months of the COVID-19 pandemic. This is $5 \%$ higher than the overall CAM use $(62.2 \%)$ reported in a similar population one year earlier [11]. In particular, it appeared that the use of natural remedies and dietary supplements had increased during the COVID-19 pandemic compared to the year before $(57.0 \%$ versus $47.4 \%$, respectively) [11]. These findings are in line with a recently published study from Poland demonstrating increased consumption of dietary supplements like vitamins $C$ and $D$, zinc, and omega-3 during the first wave of the COVID-19 pandemic [15]. In this Polish study, $13 \%$ of respondents started supplementation during the pandemic because they wanted to improve the immunity and/or to be protected against COVID-19 (13\%)[15]. In contrast to these findings, we found that a small proportion $(1.5 \%)$ of people living in Norway used natural remedies, vitamins, minerals and dietary supplements to prevent or treat COVID-19. Therefore, the observed $10 \%$ increase in supplementation in the current study cannot solely be explained by the fact that more people in Norway were using supplements to specifically prevent or treat COVID-19. Rather, this increased usage seems to be an expression of a wish to actively 
contribute to increased general well-being, to boost immunity and/or activate health-promoting pathways [12]. While supplementation increased, it became apparent that self-help practices $(24.2 \%$ versus $29.1 \%$ ) and CAM received from providers (7.8\% versus $14.2 \%$ ) had decreased compared to the year before [11]. This decrease in self-help practices and consultations with CAM provider is most likely caused by restricted access to fitness/yoga centers during the nationwide lockdown, and to the fact that CAM providers had to close down their clinics [4]. Altogether, it seems that the overall need of CAM use among Norwegian citizens has not profoundly changed by the COVID-19 pandemic. However, a shift has occurred in the type of CAM modalities used which may signify a willingness to actively participate and contribute to one's health and wellness. If one CAM modality is not accessible, people may just choose another one.

Even though consultations with CAM providers decreased by almost $50 \%$ during the first wave of the COVID-19 lockdown [11], 7.8\% of the respondents still appeared to have had consulted CAM providers during the lockdown, with good perceived effect and low risk. This finding indicates that some CAM providers managed to reorganize their practice to non-physical consultations (online/telephone consultations) [4]. Likewise, regarding the use of self-help practices, although decreased, almost a quarter of Norwegian citizens continued to use these techniques during the lockdown. Previous research has shown that practicing yoga may lower depression, anxiety, and stress, and increase general wellbeing and peace of mind during the COVID-19 lockdown [16]. Furthermore, it has been reported that mindfulness may increase well-being and provides a tool for people to deal with stressful situations such as the COVID-19 pandemic [17].

A total of $5 \%$ of the respondents reported adverse effects of the treatment. As the respondents who reported adverse effects $(n=42)$ mostly found CAM beneficial $(n=32)$, the adverse effects found in this study might have been minor and transient [18]. In addition, many patients who use CAM believe that healing crisis (you get worse before getting better) is a part of the healing process [19]. This together may also be part of the explanation why reported adverse effects from natural remedies are generally low[20]. According to Deng et al [21], patients who use CAM must make a judgement of risk versus benefit of a modality. If a CAM modality have solid evidence of effect together with low risk profile, the more likely it is that patients will use the modality. On the other hand, if the risk profile (adverse effects) is greater than the beneficial effect, then it is less likely that patients will use it. The patient must weigh the beneficial effect against risk of the modality continuously and that is what the respondents in our study seems to have done.

The current study reported that women used CAM significantly more than men. These findings are in line with previous studies on CAM use in Norway [11,22] and other studies in European countries unequivocally demonstrating that CAM use is more common in women [23-27].

\section{Limitation}

The main findings should be interpreted in light of the strengths and limitations of this study. A strength was the fact that the survey was conducted in a week's time and within a crucial, lockdown period during 
the first wave of the COVID-19 pandemic in Norway. Another strength was that a similar study was conducted in the same population one year before the outbreak of the pandemic [11]. However, the observed differences between CAM use during the first wave of the pandemic and the year before should be interpreted with the knowledge that the studies used different time frame of CAM use. In the current study CAM use within the last three months was reported, whereas this was 12 months in the study conducted one year previously [11]. This might have influenced the lower number of respondents reporting consultations with CAM providers and use of self-help practices in the current study. Another limitation of the study is that the type of adverse effects is not known. We do not know if they were mild, moderate or severe, or if it was direct or indirect adverse effects [28]. The main limitation of this study was the rather low response rate of $34.5 \%$ that might influence the generalizability of the findings. On the other hand, the fact that the sample used was stratified by sex, age and region of residence increases the representativeness of the target population. The validity of self-reported data with a recall of three months can also be questioned, though the agreement between self-reported data and registered data is generally high [29].

\section{Implication of the findings}

Despite few reports of adverse effects from natural remedies and dietary supplements, the increased use of these modalities under the COVID-19 pandemic poses further challenges regarding safe use. Encouraged by social media and online marketing, people often buy natural remedies and dietary supplements online and use them without interference of a healthcare professional [30]. Main safety concerns regarding self-use of natural remedies and dietary supplements are their quality, possible interactions with other drug-related treatments [31, 32], and contamination with toxic compounds [33]. National strategies to identify, monitor, and reduce the occurrence of adverse events associated with selfuse of CAM are therefore warranted. These strategies may include the development of an online registry system for the reporting of adverse events associated with CAM use in Norway [34], and to educate and inform consumers, patients and healthcare professionals about the potential risks associated with natural remedies and dietary supplements.

It remains to be investigated whether the observed shift toward increased use of natural remedies and dietary supplements during the first wave is maintained under the whole period of the COVID-19 pandemic. The extent to which these CAM modalities may support in the prevention of COVID-19 also remains to be investigated. A first observational study has demonstrated that supplementation with probiotics, omega-3 fatty acid, multivitamin or vitamin $D$ supplements is modest but significantly associated with a lower risk of testing positive for SARS-CoV-2 in women, but not in men [35]. However, controlled high quality studies are needed in order to confirm these results.

\section{Conclusion}

The first wave of the COVID-19 pandemic has not profoundly changed the overall need of Norwegian citizens to seek care outside the conventional healthcare system, but rather caused a shift in the type of 
CAM modalities used. The risk profile of these modalities was generally low as only $5 \%$ of the respondents reported adverse effects. Further studies are needed to confirm the change from provider based to self-administered CAM use.

\section{Abbreviations}

COVID-19: Coronavirus Disease 2019

CAM: Complementary and Alternative Medicine

I-CAM-Q: International Questionnaire to measure use of Complementary and Alternative Medicine

\section{Declarations}

\section{Ethics approval and consent to participate}

Approval was applied for at the Regional Committee for Medical and Health Research Ethics (REK 2020/133217). They concluded that the project did not fall under the definition of projects to be assessed under the Health Research Act and therefore did not need approval. Informed consent to participate was obtained from all respondents.

\section{Consent for publication}

Not applicable

\section{Availability of data and materials}

The dataset this paper has been based on has not been deposited in any repository. All dataset and materials are available from the corresponding author upon reasonable request.

\section{Competing interests}

The first author Agnete Egilsdatter Kristoffersen is a member of the editorial board (Associate Editor) of BMC Complementary Medicine and Therapies

\section{Funding}

The publication charges for this paper have been funded by a grant from the publication fund of UiT The Arctic University of Norway. No further funding was received. 


\section{Authors' contributions}

AEK and TS conceived the study and all authors compiled the questionnaire. AEK performed the initial and final analyses. AEK and TS draft the initial version of the paper and all authors reviewed subsequent versions and read and approved the final manuscript.

\section{Acknowledgements}

We want to acknowledge Ola Lillenes for administrative support in preparing the questionnaire into a computer-assisted survey, and Tine Lillegård Bergli for technical support.

\section{References}

1. Tromans S, Chester V, Harrison H, Pankhania P, Booth H, Chakraborty N: Patterns of use of secondary mental health services before and during COVID-19 lockdown: observational study. BJPsych open 2020, 6(6).

2. Cauchemez S, Kiem CT, Paireau J, Rolland P, Fontanet A: Lockdown impact on COVID-19 epidemics in regions across metropolitan France. The Lancet 2020, 396(10257):1068-1069.

3. Helse- og omsorgstjenester i nasjonalregnskapet under koronakrisen [Health and care services in the national accounts during the corona crisis] [https://www.ssb.no/nasjonalregnskap-ogkonjunkturer/artikler-og-publikasjoner/_attachment/433618?_ts=17501 caf680]

4. Stub T, Jong MC, Kristoffersen AE: The impact of COVID-19 on complementary and alternative medicine providers: a cross-sectional survey in Norway. 2020.

5. Complementary, alternative, or integrative health: What's in a name? [ https://nccih.nih.gov/health/integrative-health\#hed1]

6. Quandt SA, Verhoef MJ, Arcury TA, Lewith GT, Steinsbekk A, Kristoffersen AE, Wahner-Roedler DL, Fonnebo $\mathrm{V}$ : Development of an international questionnaire to measure use of complementary and alternative medicine (I-CAM-Q). J Altern Complement Med 2009, 15(4):331-339.

7. Kemppainen LM, Kemppainen TT, Reippainen JA, Salmenniemi ST, Vuolanto PH: Use of complementary and alternative medicine in Europe: Health-related and sociodemographic determinants. Scand J Public Health 2018, 46(4):448-455.

8. Harris PE, Cooper KL, Relton C, Thomas KJ: Prevalence of complementary and alternative medicine (CAM) use by the general population: a systematic review and update. Int J Clin Pract 2012, 66(10):924-939.

9. Nilsson J, Kallman M, Ostlund U, Holgersson G, Bergqvist M, Bergstrom S: The Use of Complementary and Alternative Medicine in Scandinavia. Anticancer Res 2016, 36(7):3243-3251.

10. Hanssen B, Grimsgaard S, Launso L, Fonnebo V, Falkenberg T, Rasmussen NK: Use of complementary and alternative medicine in the Scandinavian countries. Scand J Prim Health Care 
2005, 23(1):57-62.

11. Kristoffersen AE, Quandt SA, Stub T: Use of complementary and alternative medicine in Norway: a cross-sectional survey with a modified Norwegian version of the international questionnaire to measure use of complementary and alternative medicine (I-CAM-QN). BMC complementary medicine and therapies 2021, 21(1):1-12.

12. Seifert G, Jeitler M, Stange R, Michalsen A, Cramer H, Brinkhaus B, Esch T, Kerckhoff A, Paul A, Teut $M$ : The relevance of complementary and integrative medicine in the COVID-19 pandemic: a qualitative review of the literature. Frontiers in medicine 2020, 7:946.

13. Countries in the world by population (2020) [https://www.worldometers.info/worldpopulation/population-by-country/]

14. Kristoffersen A, van der Werf E, Stub T, Musial F, Wider B, Jong M, Wode K, Danell J-AB, Busch M, Hoenders HJR et al: Visits to Health Care Providers and use of Self-management Strategies for Prevention and Treatment of COVID-19 related Symptoms. A Population Based Cross-sectional Study in Norway, Sweden, and the Netherlands. BMC Health Serv Res 2021, In press.

15. Drywie n, ME; Zielinska-Pukos, MA Dietary Supplements during COVID-19 Outbreak. Results of Google Trends Analysis Supported by PLifeCOVID-19 Online Studies. Nutrients 2021,13, 54. In.: s Note: MDPI stays neu-tral with regard to jurisdictional claims in ...

16. Sahni PS, Singh K, Sharma N, Garg R: Yoga an effective strategy for self-management of stressrelated problems and wellbeing during COVID19 lockdown: A cross-sectional study. PloS one 2021, 16(2):e0245214.

17. Conversano C, Di Giuseppe M, Miccoli M, Ciacchini R, Gemignani A, Orrù G: Mindfulness, age and gender as protective factors against psychological distress during Covid-19 pandemic. Frontiers in psychology 2020, 11:1900.

18. Stub T, Musial F, Kristoffersen AE, Alraek T, Liu J: Adverse effects of homeopathy, what do we know? A systematic review and meta-analysis of randomized controlled trials. Complement Ther Med 2016, 26:146-163.

19. Stub T, Kristoffersen AE, Alraek T, Musial F, Steinsbekk A: Risk in homeopathy: Classification of adverse events and homeopathic aggravations-A cross sectional study among Norwegian homeopath patients. Complement Ther Med 2015, 23(4):535-543.

20. Svedlund E, Larsson M, Hägerkvist R: Spontaneously reported adverse reactions for herbal medicinal products and natural remedies in Sweden 2007-15: report from the Medical Products Agency. Drugsreal world outcomes 2017, 4(2):119-125.

21. Deng GE, Frenkel M, Cohen L, Cassileth BR, Abrams DL, Capodice JL: Evidence-Based Clinical Practice Guidelines for Integrative Oncology: Complementary Therapies and Botanicals. Soc Integr Oncol 2009, 7:85-120.

22. Kristoffersen AE, Stub T, Salamonsen A, Musial F, Hamberg K: Gender differences in prevalence and associations for use of CAM in a large population study. BMC Complement Altern Med 2014, 14:463. 
23. Wode K, Henriksson R, Sharp L, Stoltenberg A, Hok Nordberg J: Cancer patients' use of complementary and alternative medicine in Sweden: a cross-sectional study. BMC Complement Altern Med 2019, 19(1):62.

24. Bishop FL, Lewith GT: Who Uses CAM? A Narrative Review of Demographic Characteristics and Health Factors Associated with CAM Use. Evid Based Complement Alternat Med 2010, 7(1):11-28.

25. Gunnarsdottir TJ, Orlygsdottir B, Vilhjalmsson R: The use of complementary and alternative medicine in Iceland: Results from a national health survey. Scand J Public Health 2020, 48(6):602-608.

26. Molassiotis A, Fernadez-Ortega P, Pud D, Ozden G, Scott JA, Panteli V, Margulies A, Browall M, Magri $M$, Selvekerova $S$ et al: Use of complementary and alternative medicine in cancer patients: a European survey. Ann Oncol 2005, 16(4):655-663.

27. Guillaud A, Darbois N, Allenet B, Pinsault N: Predictive factors of complementary and alternative medicine use in the general population in Europe: A systematic review. Complementary therapies in medicine 2019, 42:347-354.

28. Stub T, Salamonsen A, Kristoffersen AE, Musial F: How to handle worsening of condition during treatment - risk assessment in homeopathic practice. Forsch Komplementmed 2015, 22(1):30-35.

29. Reijneveld SA, Stronks K: The validity of self-reported use of health care across socioeconomic strata: a comparison of survey and registration data. Int J Epidemiol 2001, 30(6):1407-1414.

30. Sharma V, Holmes JH, Sarkar IN: Identifying complementary and alternative medicine usage information from internet resources: A systematic review. Methods of information in medicine 2016, 55(4):322.

31. Asher GN, Corbett AH, Hawke RL: Common herbal dietary supplement-drug interactions. American family physician 2017, 96(2):101-107.

32. Jermini M, Dubois J, Rodondi P-Y, Zaman K, Buclin T, Csajka C, Orcurto A, Rothuizen LE: Complementary medicine use during cancer treatment and potential herb-drug interactions from a cross-sectional study in an academic centre. Scientific reports 2019, 9(1):1-11.

33. Ekor M: The growing use of herbal medicines: issues relating to adverse reactions and challenges in monitoring safety. Frontiers in pharmacology 2014, 4:177.

34. NAFKAM strategic plan 2021-2025 [https://nafkam.no/sites/default/files/202103/Final_210329_\%20English_NAFKAM\%20strategic\%20plan\%202021_2025\%20\%28003\%29_0.pdf]

35. Louca P, Murray B, Klaser K, Graham MS, Mazidi M, Leeming ER, Thompson E, Bowyer R, Drew DA, Nguyen LH: Dietary supplements during the COVID-19 pandemic: insights from 1.4 M users of the COVID Symptom Study app-a longitudinal app-based community survey. medRxiv 2020.

\section{Figures}


Attempt to reach: $n=7,229$

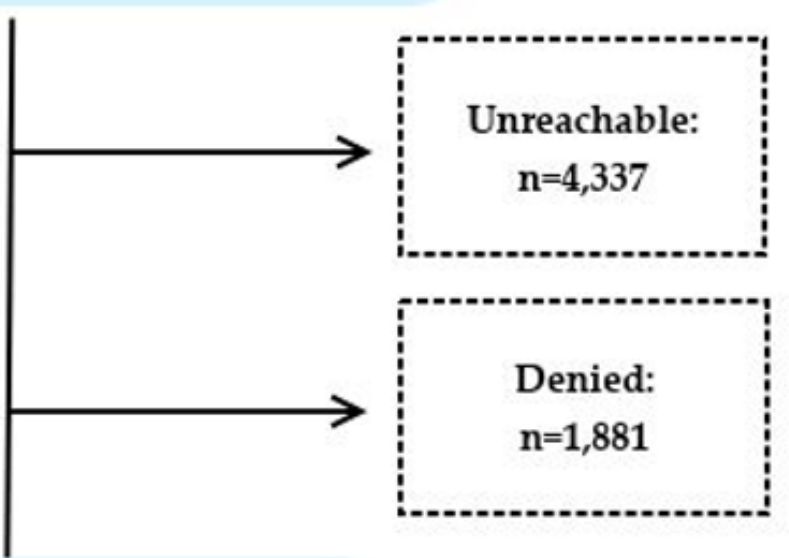

Accepted the invitation:

$n=1,011$

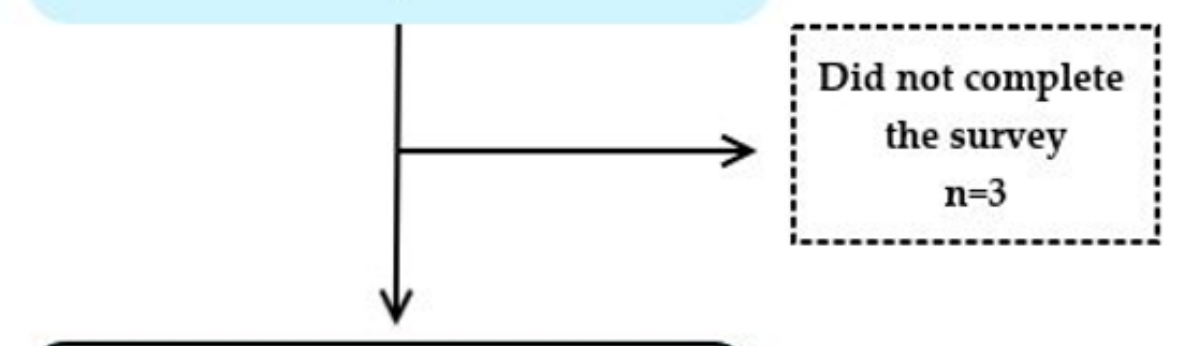

Included in the study $n=1,008$

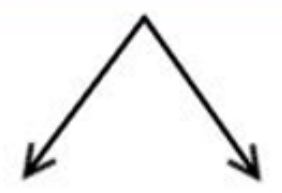

Women: $n=487$

Men: $\mathbf{n = 5 2 1}$

NAFKAM $\mid \begin{aligned} & \text { Norwajs Matiand Pasuarch Canter in } \\ & \text { Complanentary and Alematine Notione }\end{aligned}$

\section{Figure 1}

Flowchart of the respondents in the survey 